\title{
Computational Modelling of a Fluid-Conveying Flexible Channel Using oomph-lib
}

\author{
L. Lai, ${ }^{a}$ A.D. Lucey $^{\mathrm{a}}$, N.S.J. Elliott ${ }^{\mathrm{a}}$, M.D. Pitman ${ }^{\mathrm{a}}$ \\ ${ }^{a}$ Fluid Dynamics Research Group, Curtin University, GPO BOX U1987, Perth, Western Australia, 6845 \\ Email:L.Lai2@student.curtin.edu.au
}

\begin{abstract}
The objective of this paper is to assess the suitability of a new, open-source, Finite Element Modelling (FEM) program called Object-Oriented Multi-Physics Finite-Element Library (oomph-lib) to study the Fluid-Structure Interaction (FSI) mechanics of a fluid-conveying two-dimensional channel that has a flexible section. Previous studies have shown that this system contains rich dynamics that can include unstable oscillations of the flexible-wall section due to the fluid loading that itself is determined by the wall motion. The fundamental system is relevant to a host of applications in both engineered (e.g. flexible-pipes, membrane filters, and general aero-/hydro-elasticity) and biomechanical (e.g. blood flow, airway flow) systems.

The computational model developed using oomph-lib accounts for unsteady laminar flow interacting with large-amplitude (nonlinear) deformations of a thin flexible wall. The fluid loading on the wall comprises both pressure and viscous stresses while the wall mechanics includes inertial, flexural and tension forces. Nonlinear effects in the wall mechanics principally arises through the tension induced by its deformation and the correct modelling of its geometry throughout its motion. The discretised equations for the coupled fluid and structural dynamics are combined to yield a single (monolithic) matrix differential equation for all of the fluid and wall variables that is solved through a time-stepping algorithm so as to generate numerical simulations of the system behaviour.

In this paper we present results of a systematic validation of the computational model developed. Meanflow mechanics are validated by comparison against theory for Poiseuille flow through the channel with the flexible-wall held in its undisplaced position. Appropriate comparisons of statically-loaded deformations and in-vacuo vibrations of the flexible wall are made against linear theory and the limits of linear behaviour identified. The steady-state FSI is validated by comparing large-amplitude wall deformations, pressure and skin-friction loadings with published computational results that were obtained using a different computational scheme that is not in the public domain. Finally, some preliminary results of large amplitude dynamic FSI for the system are presented and discussed. Taken together, these results demonstrate the suitability of oomph-lib as a modelling and predictive tool for the study of fluid-conveying flexible pipes.
\end{abstract}

Keywords: Computational fluid dynamics, finite element analysis, fluid-structure interaction 


\section{INTRODUCTION}

The investigation regarding possible occurrence of flutter for a fluid-conveying flexible pipe with fixed ends is significant in understanding a fundamental phenomenon of Nature. It has many biomechanical applications as flexible conduits are universal in the human body. Examples of these are the arterial, venous, lymphatic, pulmonary airway and urinary systems (Bertram, 2009).

The characteristic phenomena can be reproduced in laboratory experiments using the Starling Resistor (Dodds and Runyan, 1965). A closely related model was introduced by Pedley (1992), shown in Figure 1. It consists of a 2-dimensional (2-d) channel with one segment of the wall replaced by a membrane under longitudinal tension. There are practical difficulties in producing the 2-d flow experimentally. However it has still considerable theoretical advantages as it avoids the complications of fully 3-d flows found in the Starling Resistor while exhibiting flow limitation and self-excited oscillations (Heil and Jensen, 2003).

The study of fluid-structure interaction (FSI) problems using numerical methods has become increasingly popular due to advances in computational power. The Object-Oriented Multi-Physics Finite-Element Library (oomph-lib), an open-source project, suits the objectives of this study (Heil and Hazel, 2006).

The purpose of this paper is to evaluate the suitability of oomph-1ib for 2-d flexible channel stability investigations. The scope of the present paper includes validation of the individual components and steady-state FSI of the system. Section 2 outlines the theoretical and computational modelling method used and Section 3 discusses model validation results.

\section{THEORETICAL AND COMPUTATIONAL MODELLING}

The model created is based on that by Pedley (1992). Figure 1 shows the major geometrical parameters of the model. Variables identified with asterisks are dimensional and those without asterisks are nondimensional. Fluid flow is driven by a prescribed Poiseuille velocity profile at the inlet of the 2-d channel of width $H^{*}$ and total length $L_{\text {total }}^{*}$. The total length is the summation of the upstream length $L_{\mathrm{up}}^{*}$,

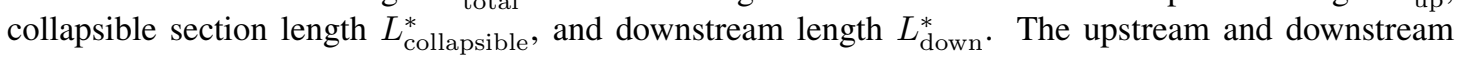
sections are rigid, and the central section is an elastic plate. The wall is loaded by an external pressure $p_{\text {ext }}^{*}$ and the traction that the fluid exerts on it.

Section 2 describes the formulation of the governing equations for the viscous fluid and flexible wall components of the oomph-1ib model. Some manipulation of these equations is performed to normalise the numerical problem so it is numerically better-conditioned. Table 1 lists the quantities used for nondimensionalisation. Quantities used here include the undeformed channel inlet pressure $p_{\text {in }}^{*}$, the dynamic viscosity $\mu^{*}$, Young's modulus $E^{*}$ and Poisson's ratio $\nu$.

Table 2 lists the four non-dimensional quantities that govern the solution space: Reynolds Number Re, Strouhal Number St, fluid pressure scale ratio $Q$ and the solid-to-fluid density ratio. Note $Q$ is the ratio of the fluid pressure scale $\mu^{*} U^{*} / H^{*}$, used to non-dimensionalise the Navier-Stokes equations (Elliott et al., 2010).

\subsection{FLUID FLOW}

The Newtonian fluid is governed by the incompressible Navier-Stokes equations. Non-dimensionalisation using the entries of Table 1 gives the Navier-Stokes equation and continuity equation in the following form

$$
\operatorname{Re}\left(\operatorname{St} \frac{\partial u_{\mathrm{i}}}{\partial t}+\mu_{\mathrm{j}} \frac{\partial u_{\mathrm{i}}}{\partial x_{\mathrm{j}}}\right)=\frac{\partial p}{\partial x_{\mathrm{i}}}+\frac{\partial u_{\mathrm{i}}}{\partial x_{\mathrm{j}}}\left(\frac{\partial u_{\mathrm{i}}}{\partial x_{\mathrm{j}}}+\frac{\partial u_{\mathrm{j}}}{\partial x_{\mathrm{i}}}\right) \quad \text { and } \quad \frac{\partial u_{\mathrm{i}}}{\partial x_{\mathrm{i}}}=0,
$$

with velocity component $u$, spatial coordinate $x$ and time $t$. Subscripts $i, j=1,2$ respectively denote the horizontal and vertical position and direction.

The velocity vector is

$$
\mathbf{V}=u_{1} \mathbf{e}_{1}+u_{2} \mathbf{e}_{2}
$$

where $u_{\mathrm{i}}$ is the velocity component and $\mathbf{e}_{\mathrm{i}}$ the unit vector; subscripts 1 and 2 respectively again denote the horizontal and vertical directions. 


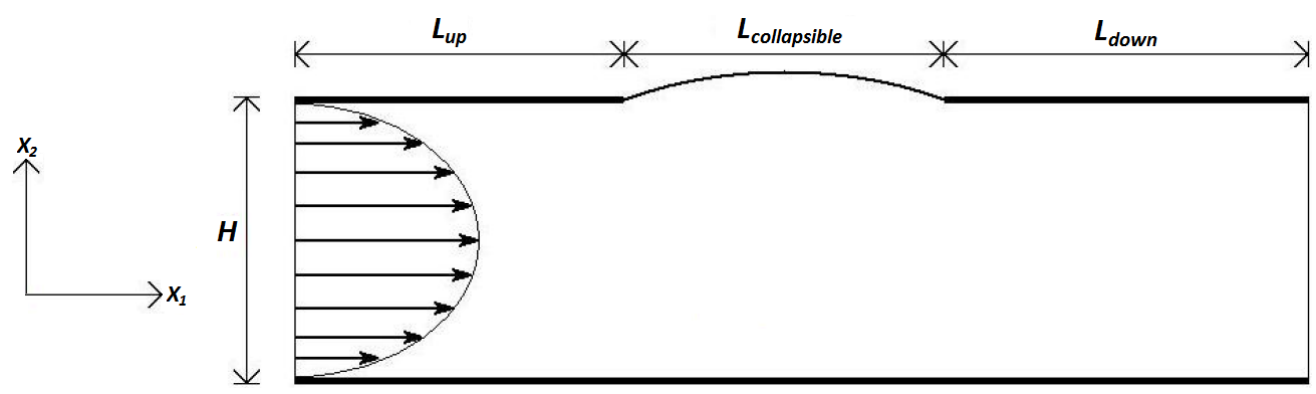

Figure 1. 2-d model of a flexible channel fixed at both ends

Table 1. Scaling quantities for non-dimensionalisation

\begin{tabular}{lll}
\hline Variable & Scaling Quantity & Mathematical Representation \\
\hline Length & Channel Width & $H^{*}$ \\
Velocity & Undeformed channel mean velocity & $V_{\text {mean }}^{*}=p_{\text {in }}^{*} H^{* 2} /\left(12 \mu^{*} L_{\text {total }}^{*}\right)$ \\
Time & Channel width / Mean velocity & $T^{*}=H^{*} / V_{\text {mean }}^{*}$ \\
Pressure & Viscous scale & $\mu^{*} V_{\text {mean }}^{*} / H^{*}$ \\
Stresses and Traction & Effective Young's modulus & $E_{\text {eff }}^{*}=E^{*} /\left(1-\nu^{2}\right)$ \\
\hline
\end{tabular}

Table 2. Solution space non-dimensional quantities

\begin{tabular}{ll}
\hline Variable & Mathematical Representation \\
\hline Reynolds Number & $\mathrm{Re}=\rho^{*} V_{\text {mean }}^{*} H^{*} / \mu^{*}$ \\
Strouhal Number & $\mathrm{St}=\left(H^{*} / V_{\text {mean }}^{*}\right) / T^{*}=1$ \\
Fluid pressure scale ratio & $Q=\mu^{*} U^{*} / E_{\mathrm{eff}}^{*} H^{*}$ \\
Solid-to-fluid density ratio & $\rho_{\mathrm{s}}^{*} / \rho_{\mathrm{f}}^{*}$ \\
\hline
\end{tabular}

The fluid flow is subject to the following boundary conditions:

- Inflow is prescribed to be a plane Poiseuille velocity profile

$$
\mathbf{V}\left(x_{1}=0, x_{2}, t=0\right)=6 x_{2}\left(1-x_{1}\right) \mathbf{e}_{1}+0 \mathbf{e}_{2} ;
$$

- No slip on rigid walls; and flexible walls.

$$
\mathbf{V}=0 ; \quad \mathbf{V}=\operatorname{St} \frac{\partial \mathbf{R}_{\mathrm{w}}}{\partial t}
$$

where $\mathbf{R}_{\mathrm{w}}$ is the flexible wall displaced position.

The finite-element model used to solve the Navier-Stoke equations discretises the fluid domain with 2d Taylor-Hood elements. Nodal positions are updated in response to the changes in the flexible-wall position when it deforms.

\subsection{FLEXIBLE WALL}

The beam elements in oomph-lib are based on geometrically nonlinear Kirchoff-Love beam theory with incrementally linear constitutive equations.

The beam's undeformed shape is parametrised by a non-dimensional Lagrangian coordinate $\xi$ and the non-dimensional position vector to a material particle on the beam's centerline in the undeformed configuration is given by $\mathbf{r}_{\mathrm{w}}(\xi)$. The unit normal to the beam's undeformed centerline is denoted by $\mathbf{n}$. The applied traction $\mathbf{f}=\mathbf{f}^{*} / E_{\text {eff }}$ (a force per unit deformed length of the beam) which deforms the beam causes its material particle to be displaced to the new position $\mathbf{R}_{\mathrm{w}}(\xi)$, and the unit normal to the beam's centerline is $\mathbf{N}$ (Elliott et al., 2010).

The non-dimensional form of the principle of virtual displacements that governs the beam deformation is then given by

$$
\int_{0}^{L}\left[(\gamma) \delta \gamma+\frac{1}{12} h^{2} \kappa \delta \kappa-\left(\frac{1}{h} \sqrt{\frac{A}{a}} \mathbf{f}+\Lambda^{2} \frac{\partial^{2} \mathbf{R}_{\mathrm{w}}}{\partial t^{2}}\right) \cdot \delta \mathbf{R}_{\mathrm{w}}\right] \sqrt{a} d \xi=0
$$


where

$$
a=\frac{\partial \mathbf{r}_{\mathrm{w}}}{\partial \xi} \cdot \frac{\partial \mathbf{r}_{\mathrm{w}}}{\partial \xi} \quad \text { and } \quad A=\frac{\partial \mathbf{R}_{\mathrm{w}}}{\partial \xi} \cdot \frac{\partial \mathbf{R}_{\mathrm{w}}}{\partial \xi}
$$

represent the squares of the lengths of infinitesimal material line elements in the undeformed and deformed configurations respectively.

We represent the curvature or the beam's centerline before and after deformation by

$$
b=\mathbf{n} \cdot \frac{\partial^{2} \mathbf{r}_{\mathrm{w}}}{\partial \xi^{2}} \quad \text { and } \quad B=\mathbf{N} \cdot \frac{\partial^{2} \mathbf{R}_{\mathrm{w}}}{\partial \xi^{2}}
$$

The strain and bending 'tensors' $\gamma$ and $\kappa$ are then given by

$$
\gamma=\frac{1}{2}(A-a) \quad \text { and } \quad \kappa=-(B-b)
$$

Next, the ratio of the natural timescale of the beam's in-plane extensional oscillations is

$$
\Lambda^{2}=\frac{H^{*}}{T_{\text {natural }}^{*}} \sqrt{\frac{\rho^{*}}{E_{\mathrm{eff}}^{*}}} \quad \text { where } \quad T_{\text {natural }}^{*}=\frac{H^{*}}{U^{*}}
$$

$\Lambda^{2}$ may be interpreted as the non-dimensional wall density, thus setting it as equal to zero corresponds to the case of zero wall inertia.

The flexible wall is discretised with one-dimensional, isoparametric, two-node Hermite beam elements.

\subsection{COUPLING AND EXTERNAL PRESSURE}

The interaction between the fluid and flexible wall is modeled using traction elements. It is assumed there is no slip for the fluid directly adjacent to the flexible wall. Thus the nodal positions of the fluid in contact with the flexible wall move in unison.

The wall is loaded by an external pressure $p_{\text {ext }}$ and the traction that the fluid exerts on it. The components of load vector $f+$ that acts on the wall are given by

$$
f_{\mathrm{i}}=-p_{\text {ext }} N_{\mathrm{i}}+Q\left(p N_{\mathrm{i}}-\left(\frac{\partial u_{\mathrm{i}}}{\partial x_{\mathrm{j}}}+\frac{\partial u_{\mathrm{j}}}{\partial x_{\mathrm{i}}}\right) N_{\mathrm{j}}\right) \quad \text { for } \quad i, j=1,2,
$$

where $N_{\mathrm{i}}$ are the Eulerian components of the outer unit normal on the boundary of the fluid domain.

\section{RESULTS OF MODEL VALIDATION}

The fluid flow component is validated in Section 3.1 and flexible wall component in Section 3.2. These components are validated separately with FSI turned off $(Q=0)$ and based on Case $u_{3}$ by Luo et al. (2008) with parameters listed in Table 3. The steady-state FSI is then validated by comparing with several other cases as listed in Luo et al. (2008) with Case $u_{3}$ being one of these. ${ }^{1}$

\subsection{FLUID FLOW}

The steady fluid component validation has been previously reported in Lai et al. (2010). A rigid channel was modelled and the pressure drop was linearly decreasing, and zero at the outlet as specified in the boundary condition. The magnitude was consistent with analytical results for a rigid channel with viscous flow. Also, the Pouseuille velocity profile specified as the inlet condition was maintained throughout the channel. A second validation was performed by applying an external pressure on the flexible wall, pushing the wall inwards. The pressure and velocity behaviour was consistent with theory again; see Lai et al. (2010) for further details.

\footnotetext{
${ }^{1}$ These parameters match that of Davies and Carpenter (1997)
} 
L. Lai et al., Computational Modelling of a Fluid-Conveying Flexible Channel

Table 3. Dimensional and Non-Dimensional Parameters

\begin{tabular}{lll}
\hline Parameter & Value & Description \\
\hline$L_{\mathrm{up}}^{*}$ & $5 \times 10^{-2} \mathrm{~m}$ & Upstream length of channel \\
$L_{\text {collapsible }}^{*}$ & $5 \times 10^{-2} \mathrm{~m}$ & Collapsible length of channel \\
$L_{\text {down }}^{*}$ & $30 \times 10^{-2} \mathrm{~m}$ & Downstream length of channel \\
$H^{*}$ & $1 \times 10^{-2} \mathrm{~m}$ & Height of channel \\
$h^{*}$ & $1.095 \times 10^{-4} \mathrm{~m}$ & Thickness of flexible wall \\
$\rho_{\mathrm{f}}^{*}$ & $1 \times 10^{3} \mathrm{~kg} \cdot \mathrm{m}^{-3}$ & Density of fluid \\
$\mu^{*}$ & $1 \times 10^{-3} \mathrm{~Pa} . \mathrm{s}$ & Dynamic viscosity of fluid \\
$\nu^{*}$ & 0.5 & Poisson's ratio of solid \\
$\rho_{\mathrm{s}}^{*} h^{*}$ & 0 & Mass per unit area of solid \\
$B^{*}$ & $7.2 \times 10^{3-9} \mathrm{~N} . \mathrm{m}$ & Flexural rigidity of flexible wall \\
$U_{\mathrm{mean}}^{*}$ & $0.02 \mathrm{~m} / \mathrm{s}$ & Inlet mean velocity \\
\hline $\operatorname{Re}$ & 200 & Reynolds Number \\
$\mathrm{St}$ & 1 & Strouhal Number \\
$\mathrm{Q}$ & $3.0429 \times 10^{-8}$ & FSI parameter \\
$\rho_{\mathrm{S}}^{*} / \rho_{\mathrm{f}}^{*}$ & 0 & Solid-to-fluid density ratio \\
\hline
\end{tabular}

\subsection{FLEXIBLE WALL}

The displacement of the flexible wall under applied traction is validated as follows. A uniform pressure is applied on the flexible wall and FSI turned off to determine the displacement of the flexible wall under external pressure effects only. This result is validated against linear Euler beam theory. The flexible wall mid point displacement from oomph-lib is divided by the theoretical value as shown in Figure 2(a). This is plotted against the external pressure applied. A 1.0 value means the theoretical and numerical results are the same. The results begin to deviate approaching an external pressure value of $1 \times 10^{4} \mathrm{~Pa}$. This deviation is due to the displacement of the flexible wall now varying from linear theory, not due to numerical inaccuracy. This is as expected as oomph-lib is a non-linear solver and has the capability to model the non-linear range.

The dynamic behaviour is validated by applying an external pressure on the flexible section that creates its Mode- 1 and Mode- 2 shapes. This external pressure is released and the flexible wall allowed to move freely. With FSI off, the beam is effectively in vacuo. This process is repeated with application of different external pressure values while maintaining the Mode-1 and Mode-2 shapes. Figure 2(b) shows the flexible wall oscillation frequency compared to theoretical values for a uniform Euler beam. The graph shows the ratio of numerical $f$ and theoretical natural frequency $f_{\mathrm{n}}$ against the flexible wall midpoint displacement. Like the static displacement results, there is good agreement with linear theory up to a certain point, then deviation occurs due to non-linearity.

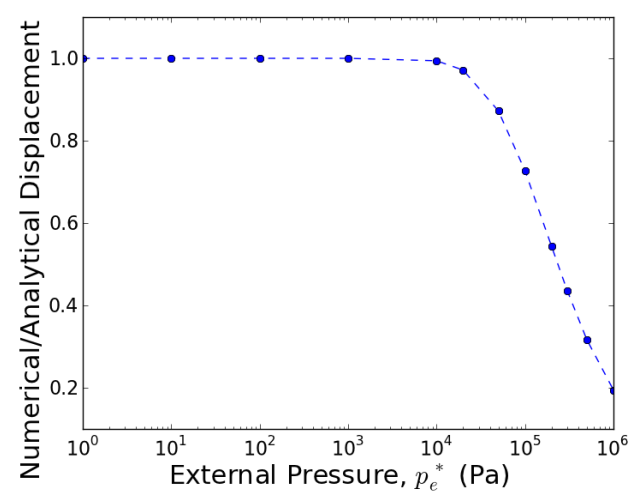

(a)

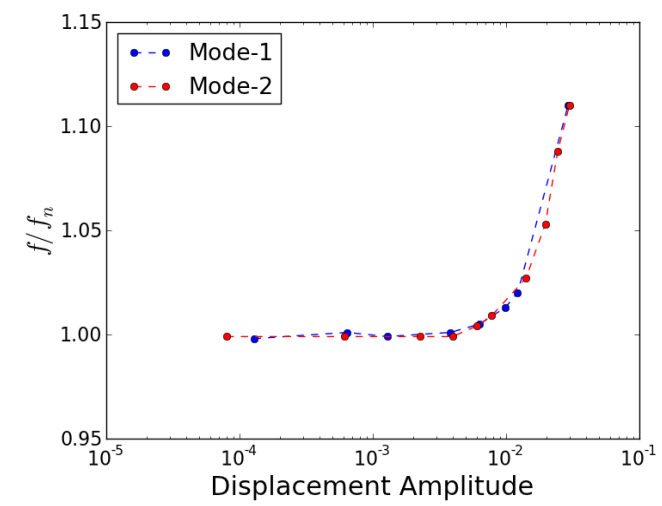

(b)

Figure 2. Flexible wall validation of the beam - convergence to linear theory. (a) Numerical-toanalytical static displacement ratio of flexible wall midpoint vs. uniform external pressure applied, and (b) Numerical-to-analytical frequency ratio vs. flexible wall midpoint displacement amplitude 


\subsection{FLUID-STRUCTURE INTERACTION}

FSI modelling is validated by comparing against Luo et al. (2008) which uses eigenvalue analysis and a different modelling scheme from oomph-lib. Figure 3(b) is the comparison of the flexible wall for different parameter values, the previously used Case $u_{3}$ was one of these cases. Figure 4 is the comparison of the fluid pressure change adjacent to the flexible wall. Both these comparisons show there is good agreement for stable FSI modelling.

In the dynamic run of these cases, an initial impulse is applied to the flexible wall external pressure. Therefore these cases are categorised based on the outcome of their dynamic runs; $s$ means the oscillation due to the impulse damps out, $n$ means the system oscillated to a steady state and continues oscillating indefinitely, and $u$ denotes a system that will oscillate with a growing displacement. Table 4 below shows the parameters scheme. The only changes to the Case $u_{3}$ parameters is in the wall stiffness value $c_{\lambda}$.

Table 4. Parameter Scheme for Luo et al. (2008)

\begin{tabular}{lccccccc}
\hline Case & $n_{10}$ & $s_{7}$ & $s_{9}$ & $s_{10}$ & $u_{1}$ & $u_{2}$ & $u_{3}$ \\
\hline Wall stiffness $c_{\lambda}$ & 1668.75 & 2400 & 1800 & 380 & 310.94 & 500 & 1000 \\
\hline
\end{tabular}

The wall stiffness is defined in Luo et al. (2008) as

$$
c_{\lambda}=\frac{E^{*} A^{*}}{\rho_{\mathrm{f}}^{*} V_{\text {mean }}^{* 2} H^{*}}
$$

where $A^{*}=h^{*} \times 1$; the flexible wall cross sectional area multiplied by the unit length.

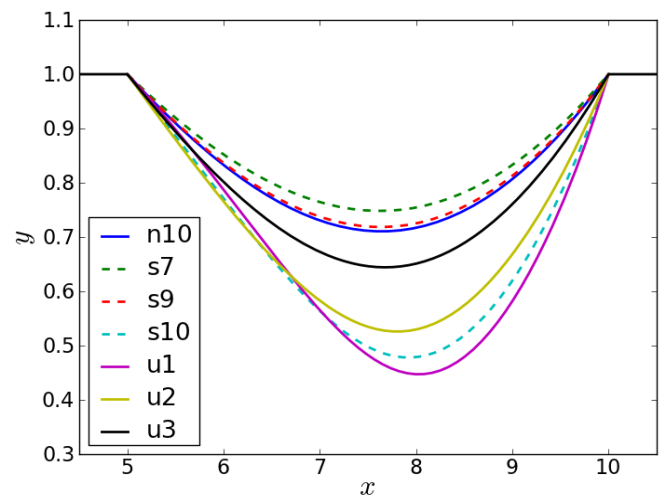

(a)

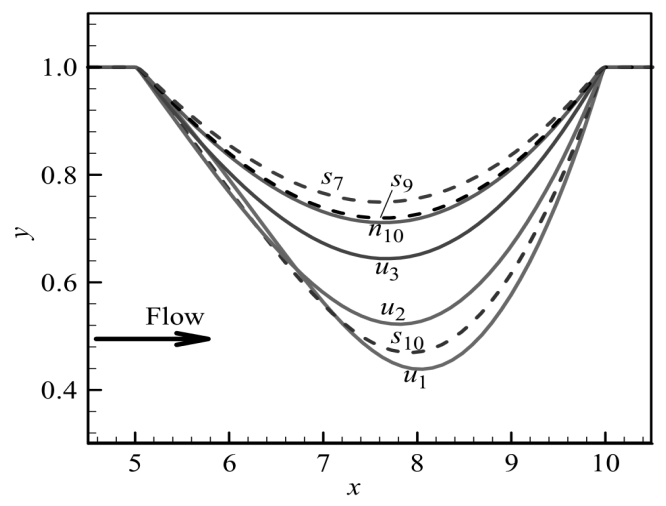

(b)

Figure 3. Displacement of flexible wall under different case parameters. (a) oomph-lib and (b) Luo et al. (2008)

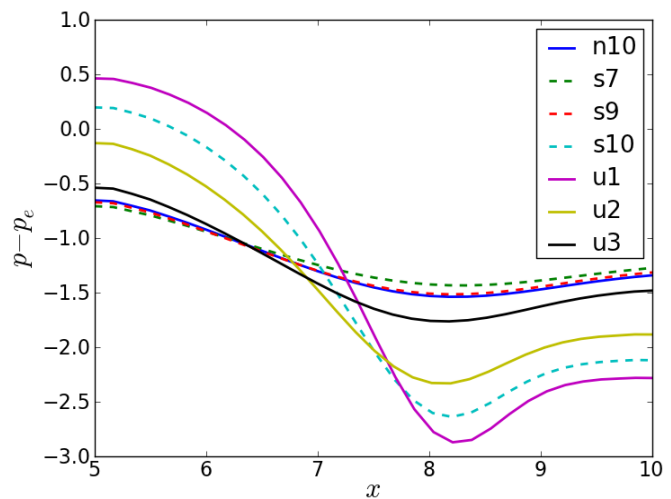

(a)

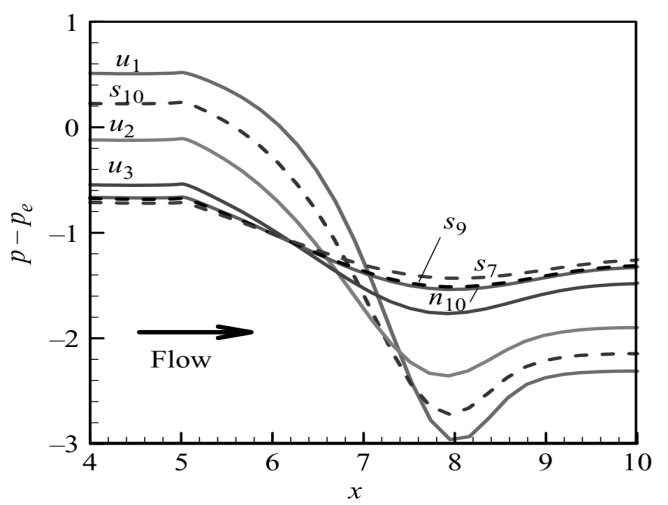

(b)

Figure 4. Flexible wall transmural pressure distribution. (a) oomph-lib and (b) Luo et al. (2008) 


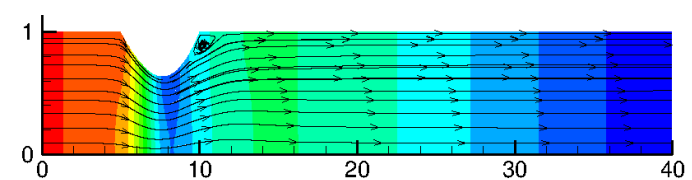

(a)

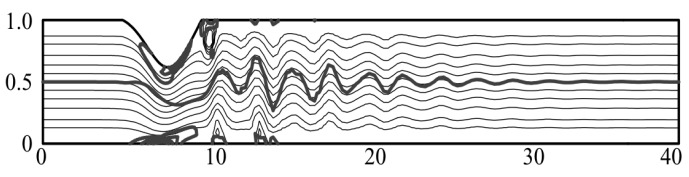

(b)

Figure 5. Fluid flow comparison. (a) oomph-lib streamlines and pressure contours (red-high, bluelow) and (b) Luo et al. (2008) streamline (thin lines) and vorticity contours (thick lines)

The fluid flow behaviour is compared for case $u_{3}$ in Figure 5. It can be seen that the fluid has oscillations occuring in the downstream section in Figure 5(b) whereas this is almost imperceptible in Figure 5(a). This is consistent with the analysis in Luo et al. (2008) which had predicted the oscillation frequency of the oomph-lib model to be significantly lower. However it is seen the behaviour of both models are consistent as there is recirculation occuring just after the flexible wall section in both models. Thus the models generate results similar except for the difference in frequency response.

\section{CONCLUSION}

This paper utilizes a new numerical scheme to study a flexible channel fixed at both ends. Study of this phenomenon has academic, industrial and biomechanical significance. The oomph-lib model is suitable for FSI study given the ease of coupling different types of physical elements. For this specific study, it enables the investigation of large displacement FSI, beyond geometrically linear limitations. The results of this paper show the fluid, flexible wall and steady-state FSI coupling validated through a comparison with the results of Luo et al. (2008).

\section{ACKNOWLEDGEMENT}

The authors would like to thank the Australian Governement through the Australian Postgraduate Award (APA) and Curtin University for their financial support.

\section{REFERENCES}

Bertram, C. (2009). Fluid flow in distensible vessels. Clinical and Experimental Pharmacology and Physiology 36, 206-216.

Davies, C. and P. Carpenter (1997). Instabilities in a plane channel flow between compliant walls. Journal of Fluid Mechanics 352, 205-243.

Dodds, H. J. and H. Runyan (1965). Effect of high-velocity fluid flow on the bending vibrations and static divergence of a simply supported pipe. NASA Technical Note NASA TN D-2870.

Elliott, N. S. J., A. D. Lucey, and M. Heil (2010, 1-5 Aug). Large-amplitude oscillations of a finitethickness cantilevered flexible plate in viscous channel flow. In Proc. ASME FEDSIM-ICNMM201030438, Montreal, Canada. ISBN 978-0-7918-3880-8.

Heil, M. and A. Hazel (2006). Oomph-lib an object-oriented multi-physics finite-element library. pp. 19-49.

Heil, M. and O. Jensen (2003). Flows in deformable tubes and channels theoretical models and biological applications. Fluid Mechanics and Its Applications 72, 15-50.

Lai, L., N. Elliott, A. Lucey, and M. Pitman (2010). Numerical two-dimensional flexible channel model fixed at both ends for flowinduced instability analysis. In Proc. 6th Australasian Congress on Applied Mechanics, Perth, Western Australia, pp. 207-211. Engineers Australia. ISBN 978-0-8582-5941-6.

Luo, X., Z. Cai, W. Li, and T. Pedley (2008). The cascade structure of linear instability in collapsible channel flows. Journal of Fluid Mechanics 600, 45-76.

Pedley, T. (1992). Longitudinal tension variation in collapsible channels: A mechanism for the breakdown of steady flow. ASME Journal of Biomechanical Engineering 114(1), 60-67. 